\section{Investigación Descriptiva, Correlacional o Cualitativa}

Pensar en Movimiento:

Revista de Ciencias del Ejercicio y la Salud ISSN 1659-4436

Vol. 10, No.1, pp. $1-15$

\section{PENSAR EN}

MOVIMIENTO

REVISTA DE CIENCIAS DEL EJERCICIO Y LA SALUD

\title{
INFLUENCIA DEL RÉGIMEN DE PRÁCTICA SOBRE LA CAPACIDAD PERCEPTUAL TÁCTIL DISCRIMINATORIA EN JUGADORES DE VOLEIBOL, BALONCESTO Y BALONMANO
}

\author{
Arián Aladro Gonzalvo, Lic. ${ }^{1(C, D, E)}$ y Gerardo Araya Vargas, M.Sc. ${ }^{1,2(B, D)}$ \\ aladro80@fastmail.es \\ ${ }^{1}$ Escuela de Educación Física y Deportes, Universidad de Costa Rica \\ ${ }^{2}$ Escuela de Ciencias del Movimiento Humano y Calidad de Vida, Universidad Nacional
}

Manuscrito recibido: 12/12/2011; aceptado: 06/06/2012; publicado: 30/06/2012

\section{RESUMEN}

Aladro-Gonzalvo, A. y Araya-Vargas, G. (2012). Influencia del régimen de práctica sobre la capacidad perceptual táctil discriminatoria en jugadores de voleibol, baloncesto y balonmano. PENSAR EN Movimiento: Revista de Ciencias del Ejercicio y la Salud, 10 (1), 1-15. Comprender los procesos cognitivos que se dan en la actividad deportiva representa un tema abierto de investigación. El propósito de este estudio fue medir, a través de un método psicofísico de campo, la capacidad perceptual táctil discriminatoria de jugadores de voleibol, baloncesto y balonmano; además, explorar si algunos indicadores relacionados con el desarrollo deportivo y el régimen de práctica predicen esta capacidad. El umbral diferencial (UD) de sensación táctil promedio durante dos mediciones fue de $20.31 \pm 8.68$ milibares $(\mathrm{mb})$ para el voleibol, $19.86 \pm 6.21(\mathrm{mb})$ para el baloncesto y $17.93 \pm 12.26$ para el balonmano. No se encontró diferencias significativas en el UD entre deportes con la misma tendencia entre mediciones. Un modelo de cinco variables predictoras explicó un $30.4 \%$ de la varianza del UD, por lo que un $69.6 \%$ se explicaría por la influencia de otras variables no contempladas en este modelo, o no controladas en el diseño del estudio. La principal contribución al porcentaje de varianza explicada del UD, correspondió a la frecuencia (días) de práctica durante la semana $(\beta=-0.42, t=-2.60 ; p=.016)$ y en segundo lugar al tiempo (horas) de práctica al día $(\beta=-0.59, \quad t=-2.99 ; p=.006)$, únicos predictores significativos del UD. De este estudio se podrían desprender aplicaciones para mejorar los procesos de aprendizaje y de rendimiento en la ejecución de los elementos técnicos deportivos, si se lograran reproducir los resultados encontrados mediante estudios causales.

Palabras claves: capacidad perceptual; percepción táctil; régimen de práctica; umbral diferencial de sensación 


\section{ABSTRACT}

Aladro-Gonzalvo, A. y Araya-Vargas, G. (2012). The Influence of Practice Regime on Tactile Perceptual Discriminative Ability in Volleyball, Basketball, and Handball Players. PENSAR EN Movimiento: Revista de Ciencias del Ejercicio y la Salud, 10 (1), 1-15. Understanding the cognitive processes that occur in sports actions represents an open question for certain research work. The purpose of this study was to assess in real-world contexts the tactile perceptual discriminative capability in volleyball, basketball and handball players, as well as to explore whether some issue related with the athletic performance and practice regime predict aforementioned capacity. The mean differential threshold (DT) of tactile sensation between two measurements was $20.31 \pm 8.68$ milibar $(\mathrm{mb}), 19.86 \pm 6.21(\mathrm{mb})$ and $17.93 \pm 12.26$ for volleyball, basketball and handball, respectively. No significant differences were found on DT among sport disciplines, with the same trend between measurements. A model of five predictor variables explained $30.4 \%$ of the DT variance; consequently a $69.6 \%$ may be explained because of the influence of other variables not included in the model, or not controlled in the study design. The mean contribution to the explained variance percentage of DT corresponds to frequency (days) of practice per week $(\beta=-0.42, t=-2.60 ; p=.016)$ and the time (hours) of practice per day $(\beta=-0.59, t=-2.99 ; p=.006)$, the only significant DT predictors. Several practical applications may be derived from the results of this study to enhance the learning process and performance of sports techniques, but whether the findings can be reproduced by means of causal studies remains to be seen.

Key Words: differential threshold of sensation; perceptual capability; practice regime; tactile perception

En la actualidad, el estudio de la percepción ha penetrado en muchas esferas de la vida cotidiana y en cada una de estas ha adquirido un contenido específico.

En el campo de la actividad física y el deporte se conoce de la existencia de una fuerte relación entre las percepciones y el dominio de nuevas formas de movimiento (Riera, 1997); tanto es así que los altos resultados en el deporte los logran aquellos atletas capaces no solo de pensar, sino también de sentir, es decir, poseen un alto nivel de posibilidades senso-perceptuales (Platonov, 2002).

También, la especialización de estas percepciones se considera de gran importancia para el desarrollo de las habilidades y hábitos motrices, pues en su génesis se observa cómo los analizadores sensoriales o los sistemas perceptivos implicados en el movimiento, captan y elaboran la información procedente del medio con una alta precisión (Lederman y Klatzky, 2009).

En este sentido, se conoce que el dominio de los complejos o nuevos gestos técnicosdeportivos está determinado por cualidades psicológicas como la sutileza de las sensaciones musculares motoras y la exactitud de las percepciones.

Los métodos psicofísicos, basándose en la determinación de umbrales, permiten cuantificar de manera precisa cuatro tipos básicos de comportamientos perceptivos: la detección, el reconocimiento, la estimación y la diferenciación (Krawczyk, 1996). 
Sin embargo, el área de investigación en psicofísica es un campo poco explorado en psicología en comparación con otros temas de estudio. A esto se adiciona que la aplicación de esta rama de la psicología experimental en el ámbito deportivo es novedosa, pues la exploración de las capacidades perceptuales que incluyen umbrales discriminatorios o diferenciales han tomado un rol mayor en aplicaciones tecnológicas como la realidad virtual, cuidados de salud, entretenimiento, entre otras. (Ino, Izumi \& Ifukube, 1999; Fujimoto, Yamakawa \& Manabe, 2000).

Por otra parte, las variables menos estudiadas en el campo de la psicofísica son las relativas al sentido háptico (tacto).

El tacto es un sistema perceptual que incorpora información de la piel (cutánea), de los músculos, tendones y articulaciones (cinestésica), los cuales generalmente se relacionan con la acción manual de exploración y reconocimiento de las propiedades de objetos tangibles (Lederman y Klatzky, 2009).

Aunque se conoce que la sensibilidad táctil parece estar determinada por la especificidad de los factores medioambientales en una disciplina deportiva dada (Kaluga y Rostkowska, 2008), los mecanismos asociados con el sistema perceptual táctil en deportistas no han sido tan investigados como las sensaciones visuales o auditivas. Por tanto, los objetivos de esta investigación fueron: determinar la capacidad perceptual táctil discriminatoria que tienen los jugadores de primera división de voleibol, baloncesto y balonmano de la Universidad de Costa Rica, comprobar si esta capacidad se comporta de manera estable entre mediciones, así como identificar si existen diferencias entre deportes y si estas variables interactúan para incrementar la capacidad perceptual. Por último, conocer cuál es la capacidad predictiva de algunas variables relacionadas con el desarrollo deportivo y el régimen de práctica sobre la capacidad perceptual táctil de estos jugadores.

\section{Metodología}

Participantes. En el estudio participaron 30 jugadores masculinos de voleibol $(n=10)$, baloncesto $(n=10)$ y balonmano $(n=10)$ de equipos de primera división de la Universidad de Costa Rica. La edad cronológica media fue 20,7 \pm 3,1 años para el voleibol, 26,4 \pm 5,3 años para el baloncesto y $25,5 \pm 3,0$ años para el balonmano.

Instrumentos. Se utilizaron 8 balones profesionales de voleibol marca Mikasa®, modelo $M V$ 210, cuyo uso está homologado por la Federación Internacional de Voleibol [FIVB, 2008]. Para comprobar los valores de presión que se modificaron en los balones, se empleó un Manómetro Digital marca Molten $\AA$, modelo $P G P$, el cual puede ser usado a temperaturas de $0-40{ }^{\circ} \mathrm{C}$ en condiciones de humedad relativa de 0-90\%, posee una mínima división de escala de 5 milibares $(\mathrm{mb})$ y la máxima presión que puede detectar es de $1200 \mathrm{mb}$. Este instrumento es utilizado y homologado por entes federativos de la FIVB por ser el dispositivo con mayor precisión en condiciones de competencia. Considerando que este instrumento tiene una mínima división de escala de $5 \mathrm{mb}$, se asumió una desviación estándar para cada medición de $\pm 5 \mathrm{mb}$.

La capacidad perceptual táctil discriminatoria se estimó mediante el umbral diferencial de sensación, al utilizar un método psicofísico de campo denominado método de los límites (Goldstein, 2011). 
Para recolectar la información del protocolo de investigación se diseñó una hoja de registro de datos (Apéndice 1). Se elaboró un cuestionario auto-administrado para obtener la información relacionada con el desarrollo deportivo y el régimen de práctica (Apéndice 2).

Procedimiento. Inicialmente se seleccionaron 8 balones nuevos de voleibol, a los cuales se les modificaron los valores de presión por medio de un manómetro digital Molten®. Estos balones se clasificaron con un sticker en la superficie para facilitar al investigador la presentación de los pares de estímulos: estímulo estándar (EE) y estímulo comparativo (EC). Además, se fijaron 9 niveles de comparación para cada par de estímulos: 240 mb, 260 mb, $280 \mathrm{mb}, 300 \mathrm{mb}, 320 \mathrm{mb}, 340 \mathrm{mb}, 360$ $\mathrm{mb}, 380 \mathrm{mb}$. El balón con el valor de $300 \mathrm{mb}$ se consideró el EE y el resto como EC, aunque también se incluyó un estímulo de igual magnitud que el estándar $(300 \mathrm{mb})$ en las series de estímulos comparativos.

El valor de $20 \mathrm{mb}$ que se manipuló durante cada comparación de estímulos se seleccionó con anterioridad durante un estudio piloto. Se estableció una presión de $300 \mathrm{mb}$ para el EE, considerando un valor prudente dentro del rango de valores de presión (294,3 a 318,82 mb) establecidos por el reglamento de la FIVB (2008) para poder utilizar los balones en eventos competitivos.

Una vez que los participantes llegaban al local destinado para desarrollar el protocolo de investigación, se les explicaba detalladamente en qué consistía la prueba y completaron en una sola ocasión el cuestionario auto-administrado sobre el desarrollo deportivo. Seguidamente, se les presentó un par de estímulos, el EE y el EC mediante varios ensayos en series aleatorizadas (ascendente y descendente). Cada vez que se mostró el par de estímulos, la tarea del participante consistió en juzgar cada EC como mayor, igual o menor al EE, de esta manera cada ensayo se detuvo cuando el participante percibió un cambio de magnitud estimular.

La presentación de cada par de estímulos se hizo de forma sucesiva y aleatoria para controlar el posible sesgo conocido como error de tiempo o espacio, el cual puede aparecer si se presenta el par de estímulos al participante siempre en el mismo orden (Goldstein, 2011). Este par se organizó en series aleatorizadas: dos ascendentes y dos descendentes. Las primeras partieron de valores inferiores al estímulo estándar $(240 \mathrm{mb})$ y las descendentes de valores superiores al estímulo estándar (380 mb), de manera tal que cada una comenzara con magnitudes estimulares diferentes, aumentando o disminuyendo los valores estimulares según el diseño preestablecido. La razón por la cual se esbozaron dos series se debió a que existían otros dos sesgos por controlar: el error de habituación y el error de expectación (Goldstein, 2011).

El error de habituación se relacionaba con la tendencia del participante a persistir en la misma respuesta, aunque de hecho se hubiese operado un cambio perceptible. Por su parte, el error de expectación se vinculó con la habilidad del participante a guiarse por el número de ensayos efectuados desde el inicio de una serie para dar su respuesta (Goldstein, 2011).

En resumen, la prueba consistió en 4 series (dos ascendentes y dos descendentes) de 8 ensayos cada una, para un número total de 32 ensayos. Las respuestas del participante se registraron en la hoja de resultados (ver Apéndice 1) con un signo de (+), (=) o (-). El tiempo de análisis y respuesta del estímulo no fue exigido ni registrado. 
Las mediciones se llevaron a cabo en un local destinado para tal efecto, de forma que los participantes concentraran toda su atención en la prueba. Cada una se desarrolló de forma individual y se le pidió a cada sujeto permanecer sentado con las manos sobre una mesa, donde se le iban colocando los balones (entiéndase como cada par de estímulos) según el orden preestablecido. Ante esto, se hace necesario aclarar que durante la aplicación del protocolo se orientó a cada sujeto cerrar los ojos en cada serie de ensayos, lo cual siempre fue comprobado por el examinador. El objetivo de esta acción consistió en aislar al sujeto de la estimulación e información visual, y que solo recibiera datos de las variaciones sistemáticas de la modalidad sensorial (tacto) estudiada, exigiendo a los participantes reaccionar con una respuesta verbal sin abrir los ojos. Esto se hizo siguiendo el mismo proceder de Kotani, Ito, Miura y Horii (2006), y con ello se intentó eliminar cualquier información visual asociada con la intensidad de la fuerza de prensión (e.g. visualizar la deformación del tejido del balón debido a la presión ejercida con las manos).

Se controló que todos los participantes desarrollaran la prueba bajo las mismas condiciones: en reposo y previo al entrenamiento; mismo local, examinador y ambiente sereno destinados para el desarrollo de dicha prueba. Los participantes la realizaron en dos ocasiones con una semana de diferencia.

Análisis estadístico. Estadística descriptiva: para la estimación del umbral diferencial, en primer lugar, se determinó el estímulo percibido como menor (EI), bajo el criterio de que fue el punto en una serie descendente donde el participante percibió el EC menor que el EE. También se estableció el valor estimular percibido como mayor (ES), el cual fue el punto en una serie ascendente donde el participante percibió el EC mayor que el EE. Seguidamente se obtuvieron los valores estimulares percibidos como mayor y menor en todas las series. La media de estos valores estimulares por cada serie divididos entre 2, representaron el UD de sensación táctil (e.g., $\mu E S-(\mu E I / 2)=U D)$.

Se obtuvo la media y la desviación estándar para la variable UD, y se estimó el porcentaje de frecuencia de las restantes variables dependientes.

Estadística inferencial: para determinar cómo se comportó el UD de sensación táctil, se realizó un ANOVA de dos vías 2 × 3 (medición x deporte) mixto. Este análisis brindó los efectos principales por factor y su interacción.

Mediante un análisis de regresión lineal múltiple y al aplicar el "Método Enter", se determinó el efecto moderador de algunas variables particulares sobre los resultados. Estas fueron codificadas a priori y se identificaron con base en los resultados de estudios previos y a la existencia de teorías que explican la relación entre las percepciones y el rendimiento deportivo. Algunas de estas variables fueron codificadas de forma categórica y posteriormente se transformaron en binarias para facilitar el análisis al aplicar la estadística inferencial paramétrica. Las variables codificadas estuvieron relacionadas con el desarrollo deportivo y el régimen de práctica de los jugadores. Desarrollo deportivo: la edad cronológica del participante fue recopilada de manera continua. La longevidad deportiva de los participantes fue codificada de forma categórica como $0=5$ años o menos y $1=$ más de 5 años. El nivel de conciencia al detectar diferencias de presión en los balones en algún momento 
de la carrera deportiva se codificó como $0=$ no he notado diferencias o nunca he pensado en ello y 1 = sí he notado diferencias.

Régimen de práctica: la frecuencia de práctica en la semana se codificó como $0=$ menos de 3 días y $1=3$ o más días. Adicionalmente el tiempo de práctica diario fue codificado en $0=2$ horas o menos y $1=$ más de 2 horas. Cabe resaltar que se tomó como criterio de práctica exclusivamente la que implicara juego o entrenamiento específico con el balón.

El análisis estadístico se realizó con el paquete SPSS® versión 15.0.

\section{Resultados}

EI UD promedio entre mediciones fue de $20.31 \pm 8.68 \mathrm{mb}$ para el voleibol, $19.86 \pm 6.21 \mathrm{mb}$ para el baloncesto y de $17.93 \pm 12.26 \mathrm{mb}$ para el balonmano. EI UD resultó inferior entre la primera (21.31 $\pm 9.12 \mathrm{mb}$ ) y la segunda medición (17.44 $\pm 9.19 \mathrm{mb}$ ), con la misma tendencia en los tres deportes (Tabla 1).

Por medio de la prueba de ANOVA se comprobó que estas diferencias no fueron significativas para los efectos en las principales mediciones $(F=2.548 ; p=.116)$ y en los deportes $(F=.363$; $p=.697$ ) (Fig. 1 y 2 ).

A pesar de que la Figura 3 mostró interacciones gráficas entre mediciones y deportes, estas no fueron estadísticamente significativas $(F=.389 ; p=.680$ ), por lo cual se asumió que el UD promedio de todas las disciplinas tuvo un comportamiento similar entre las mediciones.

En la Tabla 2 se describen las frecuencias absolutas y relativas (esta última en relación con la muestra total) correspondientes a las variables categóricas relacionadas con el desarrollo deportivo y el régimen de práctica. En la misma se puede observar que cerca de la mitad de los participantes $(n=14 ; 46.6 \%)$ indicó practicar el deporte con una frecuencia de 3 días a la semana, 5 jugadores (16.7\%) lo hacen cuatro días o más por semana, mientras que ninguno entrenaba un día por semana.

Tabla 1

Promedios y desviaciones estándar del umbral diferencial

\begin{tabular}{cccccccc}
\hline \multirow{2}{*}{$\begin{array}{c}\text { Deportes } \\
N\end{array}$} & \multicolumn{6}{c}{ UMBRAL DIFERENCIAL } \\
\cline { 2 - 7 } & \multicolumn{1}{c}{ Primera Medición } & Segunda Medición & \multicolumn{2}{c}{ Total } \\
\cline { 2 - 8 } & & $\mathrm{M}$ & $\mathrm{DE}$ & $\mathrm{M}$ & $\mathrm{DE}$ & $\mathrm{M}$ & $\mathrm{DE}$ \\
\hline Voleibol & 10 & 23.75 & \pm 9.14 & 16.87 & \pm 7.02 & 20.31 & \pm 8.68 \\
Baloncesto & 10 & 21.03 & \pm 6.99 & 18.70 & \pm 5.43 & 19.86 & \pm 6.21 \\
Balonmano & 10 & 19.12 & \pm 11.13 & 16.75 & \pm 13.80 & 17.93 & \pm 12.26 \\
\hline Total & 30 & 21.30 & \pm 9.12 & 17.44 & \pm 9.19 & 19.37 & \pm 9.28 \\
\hline
\end{tabular}

Nota: $(\mathrm{mb})$ milibares 
En relación con el tiempo de práctica al día, la mitad de los participantes $(n=15 ; 50 \%)$ indicó realizar más de 1 hora y menos de 2 horas, 3 (40\%) jugadores entrenaban más de 3 horas y ninguno lo hacía 1 hora o menos. Al mismo tiempo, un 33.3\% $(n=10)$ declaró llevar más de un año y menos de 5 practicando el deporte principal (se tomó como criterio de deporte principal, al cual el participante le dedicaba más días y horas de práctica a la semana, pues los participantes pueden practicar simultáneamente otro tipo de deporte; por su parte, el 33.3\% lo hacía por más de 10 años y tan solo el $10 \%(n=3)$ tenía menos de 1 año de práctica.

Tabla 2

Estadísticos descriptivos agrupados de indicadores de desarrollo deportivo

\begin{tabular}{|c|c|c|c|c|}
\hline \multicolumn{5}{|c|}{ Frecuencia de práctica en la semana } \\
\hline & 1 día & 2 días & 3 días & 4 o más días \\
\hline $\mathrm{n}$ & 0 & 11 & 14 & 5 \\
\hline$\%$ & 0 & 36.6 & 46.6 & 16.7 \\
\hline \multicolumn{5}{|c|}{ Tiempo diario de práctica } \\
\hline & $\leq 1 \mathrm{~h}$ & $>1-2 h$ & $>2-3 h$ & $>3 h$ \\
\hline $\mathrm{n}$ & 0 & 15 & 12 & 3 \\
\hline$\%$ & 0 & 50 & 40 & 10 \\
\hline \multicolumn{5}{|c|}{ Longevidad deportiva } \\
\hline & $\leq 1$ año & $>1-5$ años & $>5-10$ años & $>10$ años \\
\hline $\mathrm{n}$ & 3 & 10 & 7 & 10 \\
\hline$\%$ & 10 & 33.3 & 23.3 & 33.3 \\
\hline \multicolumn{5}{|c|}{ Nivel de conciencia al detectar diferencias de presión } \\
\hline & SND & & & NPE \\
\hline $\mathrm{n}$ & 22 & & & 5 \\
\hline$\%$ & 73.3 & & & 16.6 \\
\hline
\end{tabular}

Nota: SND: Si notó diferencias; NND: No notó diferencias; NPE: Nunca pensó en ello.

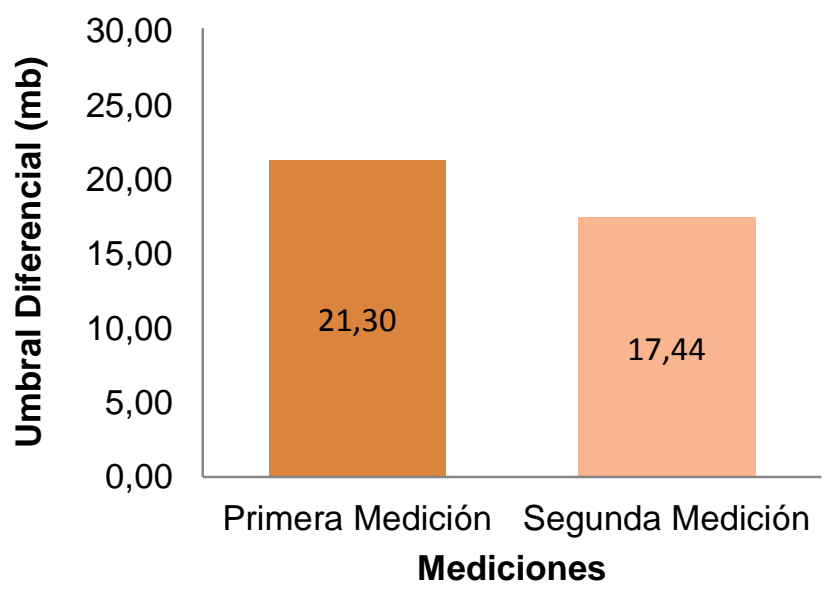

Figura 1. Comparación estadística del umbral diferencial entre mediciones. $p>.05$. 


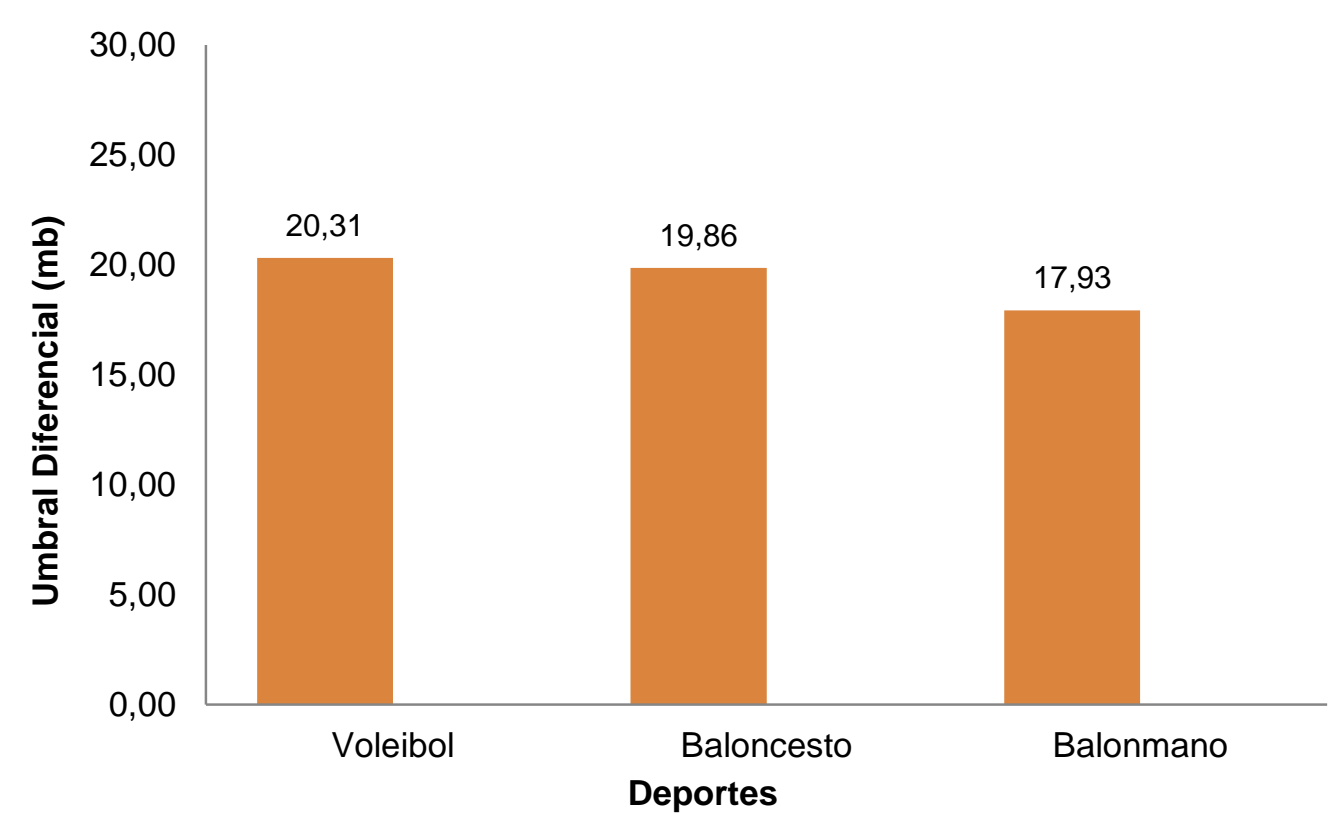

Figura 2. Comparación estadística del umbral diferencial entre deportes. $p>.05$.

En cuanto al nivel de conciencia al detectar diferencias de presión entre balones, se obtuvo que la gran mayoría ( $n=22 ; 73.3 \%)$ notó diferencias en algún momento de su carrera deportiva; 10 jugadores (33.3\%) nunca las notaron y 5 (16.6\%) nunca pensaron ello (Tabla 2).

Cabe recordar que la presión de los balones fue la única variable manipulada previo al protocolo; por tanto, los resultados del resto de las variables relacionadas con las propiedades físicas de los balones no fueron analizadas.

Por último, la edad promedio total de los jugadores fue de $24.20 \pm 4.55$ años.

Los datos mostrados en la Tabla 3, que resume los resultados del modelo de regresión utilizado, prueban que este es significativo, pues la pendiente de la línea de regresión múltiple difiere significativamente de cero $(F=3.534 ; p=.016)$. Además, al observar el coeficiente de determinación ajustada al número de variables, se aprecia que el modelo de las 5 variables predictoras explica un $30.4 \%$ de la varianza de la variable dependiente; por lo cual un $69.6 \%$ de la varianza del UD se explicaría por la influencia de otras variables no contempladas en este modelo, o no controladas en el diseño del estudio. 


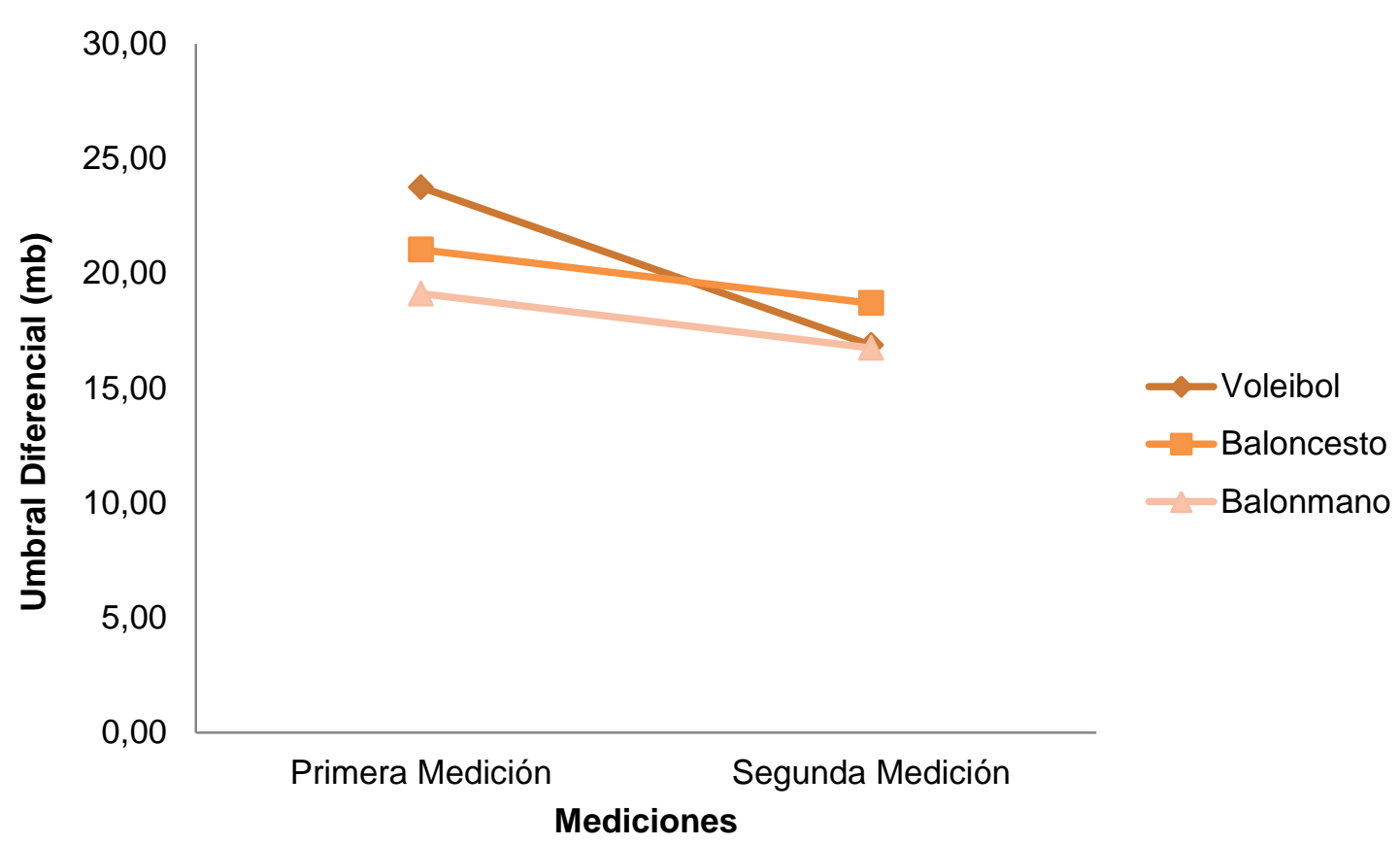

Figura 3. Umbral diferencial entre deportes y mediciones. Interacciones estadísticamente no significativas. $p>.05$.

Al revisar las correlaciones parciales presentadas en el modelo de regresión, entre las variables codificadas a priori y los puntajes promedio obtenidos del UD, se detectó que solo la frecuencia de práctica en la semana $(\beta=-0.42, t=-2.60 ; p=.016)$ y el tiempo de práctica al día ( $\beta=-0.59$, $t=-2.99 ; p=.006)$ se asociaron de manera estadísticamente significativa con el UD. Estas asociaciones ocurren de manera inversa, en donde a más días de práctica en la semana y horas de práctica al día, podrían esperarse menores valores de UD, y entre menores sean estos, mejor es la capacidad para discriminar estímulos táctiles con las manos. Variables como la longevidad deportiva y el nivel de conciencia al detectar diferencias de presión en los balones presentaron correlaciones significativas entre ellas, pero estas, para efectos de este estudio, se consideran espurias o sin sentido.

Al analizar el valor estadístico Durbin-Watson mostrado en la Tabla 3, se puede apreciar que este se encuentra cercano a 2, por lo cual se podría aseverar que los errores no están correlacionados (Visauta-Vinacua, 2002) y, por tanto, se cumple con el supuesto de independencia de los errores.

Con respecto a la colinealidad o existencia de una correlación muy elevada entre las variables independientes del modelo de regresión, con lo cual todas estas proporcionarían información similar y difícil de separar (Visauta-Vinacua, 2002); se revisó el estadístico de tolerancias y se obtuvo que 
estas oscilaron entre 0.525 y 0.930 , no encontrándose tolerancias muy bajas con respecto a las demás. Por tanto, el modelo de regresión aplicado no presentó problemas de colinealidad.

El análisis de los residuos estandarizados arrojó que el promedio $\left(6.47 \times 10^{-16}\right)$ de la distribución estuvo muy cercano a 0 y la desviación típica (0.91) a 1, con lo cual se puede asumir que el modelo de regresión cumple con el supuesto de normalidad.

También se analizó gráficamente la distribución de los residuos estandarizados, y no se apreciaron problemas de linealidad en la relación entre las variables, por no precisarse un patrón sistemático definido en los datos, y además porque los residuos estandarizados tendieron a distribuirse uniformemente entre $-2 \mathrm{y}+2$.

Tabla 3

Resumen del modelo de regresión entre algunas variables codificadas y el umbral diferencial de sensación táctil de presión

\begin{tabular}{|c|c|c|c|c|c|c|c|c|c|c|}
\hline \multirow[b]{2}{*}{ Modelo } & \multirow[b]{2}{*}{$\mathrm{R}$} & \multirow[b]{2}{*}{$\begin{array}{c}\mathrm{R} \\
\text { cuadrado }\end{array}$} & \multirow[b]{2}{*}{$\begin{array}{c}\mathrm{R} \\
\text { cuadrado } \\
\text { corregida }\end{array}$} & \multirow[b]{2}{*}{$\begin{array}{l}\text { Error tip. de } \\
\text { estimación }\end{array}$} & \multicolumn{5}{|c|}{ Estadísticos de cambio } & \multirow[b]{2}{*}{$\begin{array}{l}\text { Durbin- } \\
\text { Watson }\end{array}$} \\
\hline & & & & & $\begin{array}{c}\text { Cambio } \\
\text { en } \mathrm{R} \\
\text { cuadrado }\end{array}$ & $\begin{array}{c}\text { Cambio } \\
\text { en F }\end{array}$ & gl1 & gl2 & $\begin{array}{c}\text { Sig. } \\
\text { cambio } \\
\text { en } \mathrm{F}\end{array}$ & \\
\hline 1 & $0.651(a)$ & 0.424 & 0.304 & 6.391 & 0.424 & 3.534 & 5 & 24 & 0.016 & 1.800 \\
\hline
\end{tabular}

Nota: (a) Variables predictoras: (Constante), Edad cronológica (años), Frecuencia (días) de práctica en la semana, Tiempo (horas) de práctica al día, Longevidad deportiva (años), Nivel de conciencia al detectar diferencias de presión en los balones con las manos.

\section{Discusión}

Los resultados obtenidos muestran que no hubo diferencias significativas entre los promedios del UD de las dos mediciones realizadas, lo cual demuestra que esta capacidad se comporta de manera estable a corto plazo. Sin embargo, se presentó cierta tendencia a la reducción del UD en la segunda medición, posiblemente debido a la familiaridad con la prueba, esto sugiere que la capacidad perceptual táctil en estos deportes puede ser entrenable o afectada por varios aspectos, los cuales se intentarán explicar más adelante.

Al comparar el UD promedio de las tres disciplinas deportivas estudiadas no se encontraron diferencias significativas entre ellas. Estos resultados pueden justificar que los jugadores de voleibol no tuvieron ventaja de tipo sensorial si se comparan con los de balonmano y baloncesto que estaban menos familiarizados con las propiedades físicas de los balones utilizados en el protocolo. Además, cuando se compara el promedio total entre disciplinas, el voleibol fue el grupo que más alto umbral mostró.

A pesar de que se observaron interacciones gráficas entre las variables independientes, estas no fueron significativas, lo cual ratifica que la capacidad perceptual táctil de las tres disciplinas no resultara diferente, y se presentara con un comportamiento similar entre las mediciones. 
Cuando se exploró la influencia de algunas características consideradas moderadoras desde el punto de vista sensorial, la edad no influyó en la estimulación sensorial táctil que propiamente pudieron haber recibido los jugadores de las disciplinas estudiadas y, por tanto, no se manifestó la tendencia de que a mayor edad cronológica mayor capacidad de percepción táctil. Contrario a esto, algunas investigaciones han encontrado que los jugadores como los de balonmano asignan valores distintos a la percepción de peligrosidad del lanzamiento en función de la distancia que separa al atacante del defensor, con diferencias significativas entre las edades de los jugadores, donde los mayores muestran mejor percepción (Pérez y Lubin, 2000).

Por otra parte, la experiencia de los jugadores también parece ser un factor influyente en las percepciones. En el presente estudio, la longevidad deportiva, al ser otra característica, se vincula con la experiencia deportiva (pues, luego de realizar durante muchos años determinada actividad, la estimulación sistemática recibida aumenta la magnitud perceptual) no tuvo un efecto moderador sobre el UD de sensación táctil; sin embargo, un número considerable de estudios han encontrado resultados opuestos. Por ejemplo, Tenenbaum, Levy-Kolker, Bar-Eli y Weinberg (1994) estudiaron el papel interactivo de la experiencia de los jugadores de balonmano con la complejidad estimular, fuentes atencionales y el tiempo de exposición del estímulo sobre la percepción (recuerdo) de situaciones estructuradas de juego, y encontraron que los jugadores más experimentados recordaban las situaciones de juego con mayor precisión que quienes no experimentaron situaciones estimulares de juego más complejas.

Por su parte, Lotério-Barcelos, Pontes-Morales, Nascimento-Maciel, dos Anjos-Azevedo y Furtado-da Silva (2009) evaluaron la diferencia en el tiempo de reacción simple y de reaccióndiscriminación visual entre dos grupos (principiantes y expertos) de jugadores de voleibol; encontraron que aunque los jugadores con más tiempo de práctica (expertos) tuvieron iguales tiempos de reacción ante los estímulos visuales, estos lograron inferiores tiempos de reacción discriminativos ante estímulos distractores.

Otros resultados interesantes fueron los presentados por Ramos y Santos (2005), los cuales indican que los jugadores de baloncesto con más años de práctica mostraron un menor tiempo para tomar decisiones en contextos problemáticos de juego en comparación con aquellos de menor tiempo de práctica.

En otra especialidad deportiva, pero donde se evaluó la sutileza del tacto, Kotani et al. (2006) reportaron que el UD por arquero manifestó menos fluctuación entre las pruebas y entre los días en los deportistas expertos comparado con los de menor experiencia.

Cabe resaltar que en la mayoría de estos estudios se analizó la percepción visual, y posiblemente el efecto de la edad cronológica y la experiencia sea mayor en la especialización de ciertos sentidos y no en todos por igual.

Es posible, además, que el tiempo de estimulación sensorial (i.e., agudo y crónico) afecte de forma distinta las diferentes modalidades sensoriales. Pese a que se han desarrollado modelos capaces de explicar cuáles son los mecanismos que participan en la regulación de las funciones 
perceptuales motoras de los deportistas, no se debería pretender que el UD se comporte con estabilidad, sino como un "estado" que se valora en un determinado momento.

Se conoce que los estados son conductas o características variables en el ser humano (Weinberg y Gould, 2006), lo cual afirma que la capacidad para discriminar entre dos estímulos y detectar diferencias entre ellos, evaluada en un determinado momento, va a estar condicionada por varios procesos psíquicos y cognitivos (combinación de otros estados y de rasgos) que afectarán el proceso para integrar la información como una secuencia de eventos relacionados entre sí, donde, además, van a entrar en juego otros factores extrínsecos e intrínsecos del individuo, vinculados con su formación integral, experiencia competitiva, maduración, historia, nivel y frecuencia de la estimulación recibida del medio, así como el desarrollo y la agudeza de su sistema cognitivo, entre otros aspectos que nutren estos rasgos.

Al analizar la influencia del régimen de práctica de las tres disciplinas estudiadas sobre la capacidad perceptual táctil, los resultados mostraron que tanto la frecuencia como el tiempo de práctica moderan esta capacidad, con lo cual se refleja que a mayor cantidad de días y horas de práctica en la semana, la tendencia del UD resultó ser menor, esto indica superior capacidad sensoperceptual háptica para detectar diferencias en la presión de los balones con las manos.

Interesantemente, estos resultados también apuntan a que esta capacidad quizás es superior con un régimen de estimulación agudo y no crónico, pues se obtuvo mediante el análisis de regresión, que los jugadores con más años de práctica no muestran los menores puntajes de UD, mientras que quienes practican con más regularidad (mayor cantidad de días y horas en la semana) exhiben mejor umbral sensorial.

Por otro lado, ha sido bien documentado que el tacto tiene una papel destacado dentro de los sistemas cognitivos y las funciones cerebrales (Hagner, 2010); sin embargo, en el presente estudio se encontró que el tacto no es tan valorado por los jugadores de voleibol, baloncesto y balonmano, como en el caso de otras capacidades senso-perceptuales, como la visión o la audición.

Tanto es así, que aunque la mayoría de los jugadores (73.3\%) declararon poseer un determinado nivel de conciencia al detectar diferencias en la presión de los balones con las manos, en algún momento de su carrera deportiva, esta variable no resultó un predictor importante del UD de sensación táctil.

El motivo de la falta de relación entre estas variables pudo deberse, a que el tacto opera a nivel inconsciente, como cuando un gesto motor se ha interiorizado al punto de poder realizarlo fluidamente, es decir, sin pensar en ello.

Aún cuando existen pocos estudios disponibles que aborden la temática estudiada, pensamos que aprovechar el estudio del UD para establecer un método de entrenamiento de la capacidad atencional y de la memoria motora centrada en la capacidad perceptual táctil discriminatoria, podría tener un impacto positivo en el funcionamiento del sistema neuromuscular y, por ende, potenciar la ejecución de destrezas motoras finas, las cuales resultarían ser importantes en el manejo de 
implementos con las manos en deportes que lo requieran, dado que estas aseguran la necesaria coordinación de los movimientos precisos y sobre esta base ayudan a que el deportista asimile con más eficacia los elementos técnicos de los tipos complejos de deportes.

Este estudio tuvo un número de limitaciones. En primer lugar, durante cada ensayo no se registró el número de veces que los participantes requirieron para juzgar el EC mayor, igual o menor al EE, lo cual pudo brindar información útil relacionada con la memoria táctil (i.e., almacenamiento y recuperación de información sensorial) y su interacción con la capacidad perceptiva discriminatoria. Por otra parte, al evaluar el rendimiento en las pruebas, no se controló las posibles diferencias entre sujetos en cuanto al estado emocional, específicamente la ansiedad de estado presente en cada día de experimento. A pesar de que recientes estudios en adultos saludables han encontrado que la ansiedad auto-reportada no está relacionada con el rendimiento táctil al explorar con visibilidad limitada la forma de los objetos (Van Wijk, 2011a) incluso con diferentes barreras visuales (Van Wijk, 2011b), no sabemos cómo los efectos positivos (incremento de la activación y eficacia cognitiva) o negativos (incrementa la distracción y diminuye la función de la memoria) de este estado afectan el desempeño cuando se evalúan deportistas explorando otra propiedad física como la presión.

Finalmente, ésta línea de investigación debe extenderse con la realización de estudios causales y durante situaciones reales de juego, pues estos permitirían identificar los factores y procesos involucrados en el dominio de los gestos técnicos deportivos; además, se podrían desprender aplicaciones para mejorar los procesos de aprendizaje y rendimiento de ejecución de estos gestos, para actuar sobre aquellos indicadores modificables.

\section{Referencias}

FIVB, (2008). Rules of the games text file. Official volleyball rules approved by the 31st FIVB Congress 2008. Recuperado de http://www.fivb.org/en/volleyball/Rules/Rules.htm

Fujimoto, H., Yamakawa, S. \& Manabe, S. (2000). A measaurement of differential limen for constant force and elastic force of finger. Journal of Human Interface Society, 2(4), 67-72.

Goldstein, B. E. (2011). Sensación y percepción. Kentucky, KY Estados Unidos: Cengage Learnig, Inc.

Hagner, M. (2010). Cómo funciona la mente: la representación visual de los procesos cerebrales. ARBOR Ciencia, Pensamiento y Cultura, 743, 435-447.

Ino, S., Izumi, T. \& Ifukube, T. (1999). Desing of haptic interface sustem based on sensoru characteristics of human hand. Journal of Human Interface Society, 1(4), 9-17.Kaluga, E. y Rostkowska, E. (2008). The effect of unique environmental factors on tactile perception in athletes. Kinesiologia Slovenica, 14(1), 5-13.

Kotani, K., Ito, S., Miura, T. y Horii, K. (2006). Evaluating tactile sensitivity adaptation by measuring the differential threshold of archers. Journal of Physiological Anthropology, 26(2), 143-148.

Krawczyk, S. (1996). Psychophysical methodology and the evaluation of manual materials handling an upper extremity intensive work. En A. Bhattacharya y J. D. McGlothlin (Eds.). Occupational ergonomics: theory and applications. (pp.137-163). New York: Marcel Dekker.

Lederman, S. J. y Klatzky, R. L. (2009). Haptic perception: A tutorial. Attention, Perception, \& Psychophysics, 71(7), 1439-1459.

Lotério-Barcelos, J., Pontes-Morales, A., Nascimento-Maciel, R., Anjos-Azevedo, M. \& Furtado-da Silva, V. (2009). Time of practice: a comparative study of the motor reaction time among volleyball players. Fitness \& Performance Journal, 8(2), 103-109. 
Platonov, V. N. (2002). Teoría general del entrenamiento deportivo olímpico. Barcelona, España: Paidotribo.

Pérez, J. y Lubin, P. (2000). Estimación psicofísica de la peligrosidad del lanzamiento en balonmano. Psicothema, 12(2), 438-441.

Ramos, V. y Santos M. C. (2005). A capacidade de decisão do jovem jogador de basquetebol: um estudo com escolares em Florianópolis. [Capacidad de decisión del joven jugador de baloncesto: un estudio con escolares en Florianópolis]. Acta do Movimento Humano, 1(1), 35-40.

Riera-Riera, J. (1997). Acerca del deporte y del deportista. Revista de Psicología del Deporte, 6(1) 127-138.

Tenenbaum, G., Levy-Kolker, N., Bar-Eli, M. \& Weinberg, R. (1994). Information recall of younger and older skilled athletes: The role of display complexity, attentional resources and visual exposure duration. Journal of Sport Sciences, 12, 529-534.

Weinberg, R \& Gould, D. (2006). Foundations of sport and exercise psychology. Champaign, Illinois: Human Kinetics.

Van Wijk, C. H. (2011a). Assessing tactile perception in limited visibility could be child's play: developing the Tupperware Neuropsychological Task. South African Journal of Occupational Therapy, 41, 9-13.

Van Wijk, C. H. (2011b). Assessing tactile perception: effects of different visual barriers on performance. Perceptual and Motor Skills, 113(3), 921-928

Visauta-Vinacua, B. (2002). Análisis estadístico con SPSS para Windows ${ }^{\circledR}$. Madrid, España: McGrawHill/Interamericana de España.

Participación: A- Financiamiento B- Diseño del estudio C- Recolección de datos D- Análisis estadístico e interpretación de resultados $\mathrm{E}$ - Preparación de manuscrito.

\section{Apéndices}

Apéndice 1

Hoja de registro de datos del protocolo

\begin{tabular}{|c|c|c|c|c|}
\hline \multirow{3}{*}{ Estímulos } & \multicolumn{4}{|c|}{ Series } \\
\hline & Ascendente & Descendente & Descendente & Ascendente \\
\hline & \multicolumn{4}{|c|}{$\begin{array}{l}\text { Ensayos } \\
\end{array}$} \\
\hline $380 \mathrm{mb}$ & $\mathrm{EE}^{\mathrm{a}}$ & $E C^{b}$ & EE & EC \\
\hline $360 \mathrm{mb}$ & $\mathrm{EE}$ & $\mathrm{EC}$ & EE & EC \\
\hline $340 \mathrm{mb}$ & EC & $\mathrm{EC}$ & EC & EC \\
\hline $320 \mathrm{mb}$ & EE & EC & EE & EE \\
\hline $300 \mathrm{mb}$ & EE & EE & $\mathrm{EC}$ & $\mathrm{EC}$ \\
\hline $280 \mathrm{mb}$ & EC & EE & EC & EE \\
\hline $260 \mathrm{mb}$ & $\mathrm{EC}$ & EE & $\mathrm{EC}$ & EE \\
\hline $240 \mathrm{mb}$ & $\mathrm{EC}$ & EE & EE & EE \\
\hline
\end{tabular}

Nota: EE ${ }^{\text {a. }}$ Estímulo Estándar. EC ${ }^{\text {b }}$ Estímulo Comparativo 


\section{Apéndice 2}

Cuestionario auto-administrado sobre el desarrollo deportivo

Nombres:

Apellidos:

Edad (años):

Deporte Principal:

\section{INSTRUCCIONES}

La información que usted nos brinda hoy es muy importante para nosotros, por favor lea con detenimiento las siguientes preguntas y marca con una (X) la opción que mejor se adecúe a tu situación y experiencia.

\begin{tabular}{|c|c|c|c|c|}
\hline $\begin{array}{l}\text { ¿Cuál es su nivel de } \\
\text { competencia? }\end{array}$ & \multicolumn{2}{|c|}{ Primera División: } & \multicolumn{2}{|c|}{ Segunda División: } \\
\hline $\begin{array}{l}\text { ¿Con qué frecuencia } \\
\text { entrenas en la semana? }\end{array}$ & 1 Día: & 2 Días: & 3 Días: & 4 Días o más: \\
\hline $\begin{array}{l}\text { ¿Cuánto tiempo entrenas en } \\
\text { el día? }\end{array}$ & $\begin{array}{l}\text { Menos o igual a } \\
\text { 1horas: }\end{array}$ & $\begin{array}{l}\text { Más de } 1 \text { hora y } \\
\text { menos de } \\
\text { 2horas: }\end{array}$ & $\begin{array}{l}\text { Más de } 2 \text { horas } \\
\text { y menos de } \\
\text { 3horas: }\end{array}$ & $\begin{array}{l}\text { Más de } \\
\text { 3horas: }\end{array}$ \\
\hline $\begin{array}{l}\text { ¿Cuántos años desde que } \\
\text { usted comenzó la práctica } \\
\text { sistemática o de rendimiento } \\
\text { en el deporte al cual le } \\
\text { dedica más horas de práctica } \\
\text { a la semana? }\end{array}$ & $\begin{array}{l}\text { Menos o igual a } \\
1 \text { año: }\end{array}$ & $\begin{array}{l}\text { Más de } 1 \text { año y } \\
\text { menos de } 5 \\
\text { años: }\end{array}$ & $\begin{array}{l}\text { Más de } 5 \text { años } \\
\text { y menos de } 10 \\
\text { años: }\end{array}$ & $\begin{array}{l}\text { Más de } 10 \\
\text { años: }\end{array}$ \\
\hline \multicolumn{5}{|c|}{$\begin{array}{c}\text { ¿En algún momento de su carrera deportiva ha sentido que puede notar diferencias entre balones } \\
\text { aparentemente iguales, en cuanto a?: }\end{array}$} \\
\hline & Peso & Textura & Tamaño & Presión \\
\hline \multicolumn{5}{|l|}{ Si he notado diferencias } \\
\hline \multicolumn{5}{|l|}{ No he notado diferencias } \\
\hline Nunca he pensado en ello & & & & \\
\hline
\end{tabular}

Nota: Deporte principal: actividad a la cual dedica la mayor cantidad de horas de práctica en la semana.

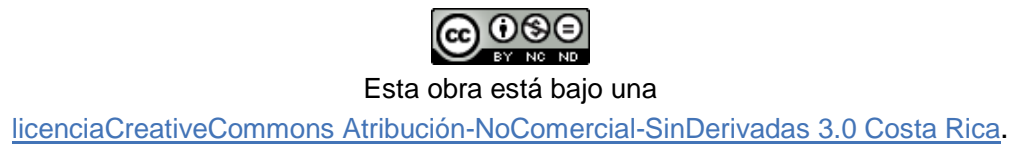

